\title{
Activity-based neuronal labeling
}

Methods to image the ongoing activity of neurons abound, with most relying on changes in cytosolic calcium levels as a proxy for neural activity. However, tools to mark and manipulate previously activated neurons within tightly controlled temporal windows are still limited, despite their potential to help study the circuit-level mechanisms of a diverse array of cognitive behaviors. New research published in Nature Biotechnology (doi:10.1038/nbt.3909; published online 26 June 2017) provides a novel and highly flexible tool for scientists to link expression of a desired transgene to neurons previously activated during defined time-frames.

Building on previous work to develop molecular switches that relied on rises in neuronal calcium levels combined with simultaneous photostimulation, the team
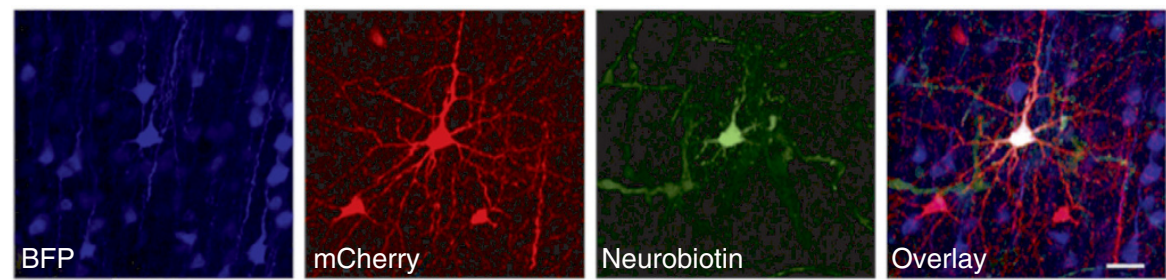

Example of a previously activated neuron successfully labeled with FLARE system in vivo. ChrimsonmCherry was induced by FLARE in vivo, with neurobiotin injected after patch-clamp slice physiology. Adapted from Nat. Biotechnol. doi:10.1038/nbt.3909; published online 26 June 2017.

used 'directed evolution' to build a significantly improved light-and-calcium gated labeling system, which they call FLARE (for Fast Light- and Activity-Regulated Expression). Data using cell cultures demonstrated significant improvements for monitoring calcium-with-light changes in transgene expression using FLARE. The group also showed that FLARE can be used to both report and manipulate previously active neurons in vivo using natural stimulation (wheel running-based activation of motor cortex neurons), although their results indicate further enhancements to FLARE will be necessary to improve signal-to-noise ratios. Dustin M. Graham 